Revista de Psicología Vol. 36 (1), 2018 (ISSN 0254-9247)

\title{
Validación convergente de un auto informe de valores prototípicos del trastorno histriónico de la personalidad
}

\author{
Leonardo Yovany Álvarez Ramírez ${ }^{1}$ \\ Corporación Universitaria de Investigación y Desarrollo, Colombia
}

Se estudió la validez convergente de la identificación que los participantes de dos grupos mixtos equivalentes, -uno de casos y otro de control-, realizó sobre un repertorio de valores prototípicos del trastorno histriónico de la personalidad, teniendo en cuenta su importancia en la difusión y desliteración en la Terapia de Aceptación y Compromiso ACT. Se aplicó la entrevista SCID-II, el Inventario Multiaxial de la Personalidad MCMI-III y una entrevista estructurada. Tras el análisis de contenido de las entrevistas estructuradas y del auto reporte (A-R-VP) de identificaciones con los valores del trastorno en cuestión, se halló que los participantes con el trastorno histriónico de la personalidad arrojaron las medias más altas en una escala de $0-5$, con valores más cercanos a 5 , mientras que aquellos sin dicho trastorno mostraron puntajes de identificación entre 0 y 1 en la misma escala. Los resultados de la prueba $t$ de student confirmaron lo anterior. Se concluye que las personas evaluadas con trastorno histriónico de la personalidad se identifican con un repertorio de doce valores característicos de este trastorno.

Palabras clave: Trastorno, histriónico, personalidad, ACT.

\section{Prototypical values self-report in the avoidant personality disorder}

This study conducted convergent validation on participant's identification with the prototypical values of histrionic personality disorder repertoire, an important part of acceptance and commitment psychotherapy (ACT). The Multiaxial Inventory of Personality (MCMI - III) was administered to two mixed groups of participants (cases versus controls). Participants were also interviewed using the SCID- II, and a structured interview The content analysis of the structured interviews showed that participants with the disorder had the highest mean in the "A-R-VP self-report identification" at all the 12 values examined on a 0 to 5 point scale, with scores closer to 5 . Meanwhile, those without the disorder had scores of identification between 0 and 1 . The results of the Student $t$ test confirmed the findings.

1 Especialista en Desarrollo Intelectual y Educación de la Universidad Autónoma de Bucaramanga (Colombia). Docente-investigador del Programa de Psicología de la Corporación Universitaria de Investigación y Desarrollo. Dirección postal: Calle 9 No. 23-55. Bucaramanga, Colombia. Contacto: leonardoalvarez64@gmail.com. 
People with histrionic personality disorder identify themselves with a repertoire of twelve characteristic values of this disorder.

Keywords: Disorder; histrionic; personality, ACT.

Validaçáo convergente de um auto-relato de valores prototípicos de transtorno de personalidade histriônica

O presente estudo validou a identificação com um repertório de valores prototípicos do transtorno de personalidade histriônica de um grupo de participantes com esse distúrbio em oposição a outro grupo de participantes sem este trastorno de personalidade que são importantes na defusão e na deliteração no Terapia de aceitação e compromisso (ACT). Desta forma, formaram-se um grupo de casos e outro grupo de controles. Ambos os grupos foram misturados. A entrevista SCID-II, o Inventário de Personalidade Multiaxial MCMI-III e a entrevista estruturada foram aplicadas. Os participantes com o transtorno tiveram os meios mais elevados em seu relatório "A-R-VP de identificação com um conjunto de 12 valores con escores perto de 5 , examinados em uma escala de 0 a 5 , em oposição àqueles que não tinham o referido transtorno, que apresentavam escores entre 0 e 1 . Os resultados do teste de Student t confirmaram o acima. Conclui-se que as pessoas avaliadas com transtorno de personalidade histriônica são identificadas com um repertório de doze valores característicos desta desordem.

Palavras-chave: Desordem, histriónico, personalidade, ACT.

Validation convergente d'un auto-rapport des valeurs prototypiques du trouble de la personnalité histrionique.

La présente étude a validé de manière convergente l'identification que les participants de deux groupes mixtes équivalents, l'un de cas et l'autre de contrôles, ont fait sur un répertoire de valeurs prototypiques du trouble de personnalité histrionique, en tenant compte de son importance dans la diffusion et l'acceptation dans la thérapie d'acceptation et d'engagement (ACT). L'interview SCID-II, l'Inventaire Multiaxial de Personnalité MCMI-III et une entretien structurée ont été appliquées. Après avoir analysé le contenu des entretiens structurés et l'auto-rapport (A-R-VP) des identifications avec les valeurs du trouble en question, il a été constaté que les participants avec le trouble de la personnalité histrionique montraient les moyens les plus élevés sur une échelle de $0-5$, avec des valeurs plus proches de 5 , alors que celles sans trouble présentaient des scores d'identification entre 0 et 1 sur la même échelle. Les résultats du test t de Student ont confirmé ce qui précède. On conclut que les personnes évaluées avec un trouble de la personnalité histrionique sont identifiées avec un répertoire de douze valeurs caractéristiques de ce trouble.

Mots-clés: trouble, histrionique, personnalité, ACT. 
La Terapia de Aceptación y Compromiso (ACT) es una terapia que dimensiona a la persona en su contexto y a partir de allí la lleva por los diferentes componentes del proceso terapéutico: la aceptación, defusión, la conciencia en el aquí — ahora, los valores y la acción, todo, con el fin de recontextualizar su comportamiento de una manera unificada y flexible (Hayes, Strosahl \& Wilson, 1999). Una de las principales fortalezas de la ACT es que se funda en un modelo y en un programa de ciencia básica (Hayes, Barnes-Holmes \& Roche, 2001), que soporta sus técnicas (Hayes et al., 1999; Luoma, Hayes \& Walser, 2007) y hace que la intervención terapéutica y los procesos clínicos, aporten resultados demostrables (Hayes, Luoma, Bond, Masuda \& Lillis, 2006), en trastornos emocionales, el abuso de sustancias o los desórdenes de la alimentación (Gallagher, 2009).

La utilización de la ACT ha sido exitosa en diversos problemas como por ejemplo, en las enfermedades crónicas (Wicksell, Renofalt, Olsson, Bond \& Melin, 2008), en problemas con adolescentes (Greco, Lambert $\&$ Baer, 2008), en problemas de depresión (Addis \& Jacobson, 1996), en el abuso infantil (Marx \& Sloan, 2002; Rosenthal, Rasmussen-Hall, Palm, Batten \& Follette, 2005); en la reducción del distrés (Kashdan, Barrios, Forsyth y Steger, 2006), en el consumo adictivo de marihuana (Twohig, Shoenberger \& Hayes, 2007), en la depresión (Zettle, 2007; Zettle, Rains \& Hayes, 2011), en la adicción al cigarrillo (Gifford et al., 2004), en el dolor crónico (Dahl et al., 2005; Wicksell, Ahlqvist, Bring, Melin \& Olsson, 2008), en la ira (Eifert, McKay \& Forsyth, 2006), en el manejo de la diabetes (Gregg, Callaghan, Hayes y Glenn-Lawson, 2007), en la ansiedad (Eifert \& Forthsyte, 2005), en el trauma (Follette y Pistorello, 2007), en la psicosis (Bach \& Hayes, 2002), en el trastorno obsesivo-compulsivo (Twohig et al.,2010), en el estrés laboral (Bond \& Bunce,2000), en el desarrollo de habilidades de empatía y cuidado de los demás (Villatte, Monestès, McHugh, Freixa i Baqué \& Loas, 2008). 
Si bien, la aplicación de la ACT ha sido eficaz en los diferentes trastornos antes mencionados, aún no se tiene noticia de su aplicación y resultados en los trastornos de la personalidad, entre ellos el histriónico, lo cual se convierte en un vacío empírico, teórico y práctico en el contexto clínico que quizás subsiste por la baja prevalencia de este trastorno en la población mundial y quizás por la desafección de los investigadores por creer en su evolución clínica insatisfactoria respecto de otros trastornos existentes (Esbec \& Echeburúa, 2011). El THP se define por la excesiva emocionalidad y la tendencia a llamar la atención y el DSM-IV y el DSM-V lo definen a través de: la erotización de las interacciones, emocionalidad cambiante y exagerada, exhibicionismo, expresión teatral, sugestionabilidad,exagerada percepción de intimidad con los demás.

Por otra parte, dentro de los instrumentos de evaluación de los trastornos de la personalidad (TP), entre ellos el histriónico, existe el índice de fiabilidad diagnóstica de las entrevistas estructuradas tales como el SCID-II (Spitzer, Williams \& Gibbon, 1989), también existe apoyo al uso de la psicobiografía y la historia clínica basadas en conductas y no en rasgos o disposiciones del paciente y por ello el aval para el uso del DSM y del CIE-10, así como el Examen Internacional para los TP (López-Ibor,Pérez,Urdanis \& Rubio,1996; Skodol et al., 2011), que se enfoca en el grado de deterioro en el funcionamiento interpersonal e intrapersonal y su severidad en el TP (Schmeck et al., 2013). Se ha utilizado con relativo éxito el Inventario de Temperamento y Carácter revisado (TCI-R) (Cloninger, 2000) en su versión adaptada al idioma Castellano por Gutiérrez-Zotes et al. (2004), el test de Empatía Cognitiva y Afectiva (TECA) (López-Pérez, Fernández-Pinto \& Abad, 2008) así como el MMPI-2-RF y el MCMI-III (Inventario Clínico Multiaxial de Millon (Millon, Millon, Davis y Grossman, 2006; adaptación española de Cardenal \& Sánchez, 2007).

Si bien son los cuestionarios los instrumentos a los cuales más se recurre en la clínica y la investigación psicológicas (Esbec \& Robles, 2013) son muy vulnerables a la manipulación por la falta de motivación, la baja conciencia del problema y la simulación y la deseabilidad social (Echeburúa, Fernández Montalvo \& Amor, 2006). Desde esta perspectiva la 
entrevista clínica en el TP se destaca por cuanto puede evaluar de manera multicontextual y multifuente (familiares, pareja, hermanos, vecinos, amigos) información colateral que permite ser verificada (FernándezMontalvo \& Echeburrúa, 2008). Con todo, la utilización conjunta de cuestionarios, entrevistas estructuradas, junto con auto informes en el caso de los TP son recomendables (Esbec \& Echeburrúa, 2014).

En el caso específico de la ACT no se cuenta con un instrumento para examinar los valores prototípicos de clientes con el trastorno en cuestión. El lugar de un auto informe de valores prototípicos del trastorno histriónico de la personalidad toma valor en cuanto instrumento para evaluar el repertorio de valores importantes para el paciente y de esta manera guiar la conciencia de los mismos en él y aparte de ello ofrecer una ruta expedita para la intervención, especialmente en la etapa de defusión y desliteración en las cuales se precisa de re-evaluar todo un sistema de valores personales que define las motivaciones del consultante en su vida, así como revela sus vulnerabilidades (Cohen, García, Apfel \& Master, 2006). Muchos estudios revelan que la ACT utiliza protocolos diferentes respecto del nivel de la técnica frente a los trastornos que aborda; sin embargo, los trastornos de la personalidad no han sido documentados con intervenciones de la ACT, lo cual es un problema, ya que estos son prevalentes en la población en general.

En concreto, algunos autores en la ACT han introducido listas de valores posibles de las cuales el terapeuta podría seleccionar aquellos que se avinieran al trastorno del consultante (Addy, 2013), sin embargo el uso de un instrumento más formal y específico para identificar ese posible repertorio prototípico de valores propio de cada trastorno de la personalidad y en especial del histriónico es aún inexistente. Como se comentó antes, los valores en este modelo terapéutico son abordados como orientaciones hacia la acción pero la importancia de delimitar los más relevantes es capital en términos de claridad terapéutica y eficacia.

A partir de lo anterior, se observa una necesidad de contar con un instrumento válido de tipo auto-informe que permita identificar dicho repertorio de valores prototípicos en el cliente con trastorno histriónico de la personalidad. 


\section{Método}

Se llevó a cabo un estudio de validación convergente de un repertorio prototípico de valores del trastorno histriónico de la personalidad en un diseño expost-facto de casos y controles.

\section{Participantes}

Para el estudio fueron seleccionados por conveniencia de la población general 149 participantes, de quienes se seleccionaron por pareamiento 120, 60 de ellos de cada género (30 mujeres y 30 hombres) con trastorno histriónico de la personalidad según el $\mathrm{DSM}-\mathrm{V}^{\oplus}$; $\mathrm{y}$, otros 60 (30 hombres y 30 mujeres) sin dicho trastorno de la personalidad, pero con edades, género, nivel educativo, estrato socioeconómico y estado civil equivalentes para establecer los respectivos grupos de casos y controles.

La fuente de participantes seleccionados fue una base de datos en la cual se registraron personas interesadas en el estudio, el cual se divulgó a través de correo electrónico, redes sociales, voz a voz, emisora universitaria, entre otros medios, previa entrevista individual. En esta se aplicó a todos los participantes el Inventario Multiaxial de Personalidad MCMI-III de Theodore Millon, en su edición española de 2007 (Cardenal \& Sánchez, 2007). Con respecto al grupo de los casos con personalidad histriónica, se seleccionaron aquellos que presentaron tasa base superior a 85 (Weltzler, 1990) y, para los controles, puntuaciones (TB) entre 0 y 15 (Cardenal \& Sánchez, 2007; Millon \& Davis, 1998). En los dos grupos (casos y controles) se observó el índice de sinceridad $34<\mathrm{X}<178$. Las edades de los participantes estuvieron entre 28 y 33 años, con estudios profesionales, del estrato socio económico 3 y de estado civil casado, de la ciudad de Bucaramanga (Colombia). Estos criterios sociodemográficos se derivaron de lo establecido en Colombia por la ley 142 de 1994 y por el Conpes 3386 (Departamento Nacional de Planeación de Colombia (2005), así como por el DANE (Departamento Nacional de Estadísticas de Colombia (2011). 


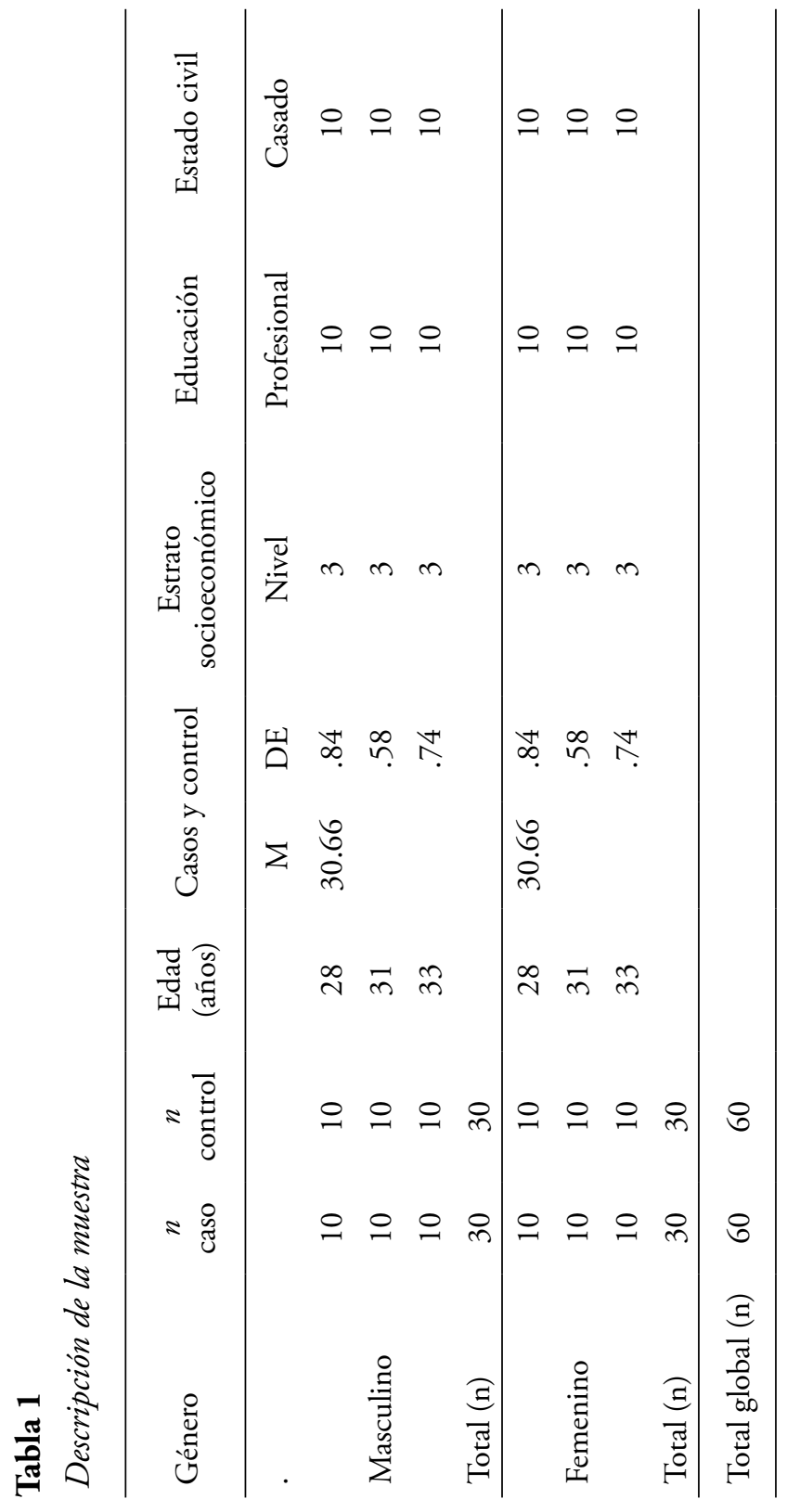




\section{Medición}

El Inventario Multiaxial de Personalidad MCMI-III (Cardenal \& Sánchez, 2007; Cardenal, Sánchez \& Ortiz-Tallo, 2007) en su versión española se utilizó para clasificar el grupo de los casos de los controles. Este instrumento evalúa la gravedad de la entidad psicopatológica, desde moderada a acentuada, a través de 175 ítems de respuesta dicotómica (falso/verdadero), contiene escalas de validez y de tendencia de respuesta; su baremación por sexo consiste en puntuaciones directas (PD) y tasas base (TB). Su confiabilidad está en $\alpha=.80$ y el test-retest fue de $\alpha=.84$ y $\alpha=.96$. Los rasgos de personalidad clínicos se ubican entre los 75 y 85 puntos y las de trastorno propiamente dicho, superiores a 85 . Posee un índice de sinceridad que invalida la prueba con puntajes menores a 34 y superiores a 178. Su tiempo de aplicación oscila entre los 15 y los 25 minutos.

La SCID II (Entrevista clinica estructurada para los trastornos de la personalidad del eje II del DSM-IV . Guia del usuario. Entrevista clínica. Cuestionario de personalidad (First, 1999, 2001; Frances, Pincus y First, 2008) se utilizó para seleccionar los casos y los controles del estudio. Se trata de una entrevista semi estructurada diagnóstica para la evaluación de 12 trastornos de la personalidad del Eje II del DSM IV-TR ${ }^{\oplus}$ que permite un diagnóstico tanto dimensional (cantidad de criterios codificados para el trastorno de la personalidad) como categorial (ausente o presente).

La entrevista considera en forma sucesiva 10 trastornos de personalidad específicos y 2 categorías del apéndice; también consta de preguntas y criterios del DSM-IV-TR para el diagnóstico; las puntuaciones de los ítems criterio de trastorno de la personalidad son: ?, 1, 2, 3, los cuales corresponden a: "información inadecuada para codificar el criterio como 1, 2, 3; luego, el valor 1, que equivale a "ausente o falso"; el valor 2 , a un nivel "sub umbral", es decir "casi se alcanza el nivel, o, el rasgo se halla presente pero sin la gravedad suficiente como para causar deterioro o malestar; y luego, el valor de 3, que significa "umbral o verdadero": patológico, persistente y generalizado (Frances, Pincus \& First, 2008). En caso de que se cumplan los criterios de más 
de un trastorno de personalidad, el entrevistador señala el "diagnóstico principal del Eje II". El entrevistado es la fuente principal de información, sin embargo, hay que tener en cuenta información de terapeutas anteriores, miembros de la familia u otros.

Por otra parte, se utilizó una entrevista estructurada como instrumento para obtener información descriptiva respecto de los casos con trastorno histriónico de la personalidad vs los controles con miras a generar un análisis de contenido que permitiera derivar un set de valores comunes en los grupos "caso" (Ver apéndice 1). El acuerdo interjueces con respecto a la estructura de la entrevista (psicólogos profesionales docentes universitarios) tuvo un coeficiente de Kappa $=.78$

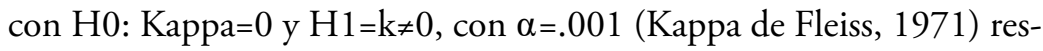
pecto de indicadores de saturación de contenido, extensión, sintaxis, las cuales calificaron en una escala de 0 a 5 donde 0 es el menor valor y 5 el mayor.

\section{Procedimiento}

\section{Primera etapa}

Se obtuvo la aprobación del protocolo del procedimiento por parte del Comité de Ética de la Corporación Universitaria de Investigación y Desarrollo-Bucaramanga (Colombia) para la realización del estudio. Se reunió un número inicial de 356 participantes (incluidos los dos géneros), candidatos potenciales elegibles para el estudio, quienes voluntariamente, previa explicación del estudio, su objetivo, procedimientos y alcances, se registraron en la base de datos. El estudio se dio a conocer a través de correos electrónicos, redes sociales, emisora universitaria, voz a voz de los estudiantes del programa de psicología, visitas a las Juntas de Acción Comunal de algunas comunidades urbanas, entre otras.

\section{Segunda etapa}

Luego de revisar la base de datos se eliminaron aquellos registros que tenían datos incompletos del participante o que al ser contactado por teléfono o vía electrónica no respondió o lo hizo para expresar su 
dimisión de participación en el estudio. Con los que quedaron en la base confirmados, se realizó una agenda de programación de entrevistas individuales con cada candidato realizadas por estudiantes de últimos semestres del programa de psicología, los cuales fueron entrenados para dicho propósito y fueron escogidos para el estudio, previa evaluación de su desempeńo por tres docentes-investigadores que se asignaron a esta etapa del estudio.

A cada participante se le aplicó en la entrevista La SCID II (Entrevista clínica estructurada para los trastornos de la personalidad del eje II del DSM-IV-TR. Guia del usuario. Entrevista clinica. Cuestionario de personalidad. (First, 2001; Frances, Pincus y First, 2008) para el diagnóstico del trastorno histriónico de la personalidad, previa lectura, explicación y firma del consentimiento informado APA en el cual se detalló los procedimientos a realizar, objetivos y alcances del estudio, regla de confidencialidad y política de transparencia, devolución de resultados en sesión individual, entre otros. Cumplieron los criterios diagnósticos del trastorno histriónico de la personalidad 37 hombres y 39 mujeres, pero se incluyeron los datos de 30 de cada género debido a que algunos se retiraron, dieron información inconsistente o fueron poco cooperativos para adelantarles los procedimientos.

\section{Tercera etapa}

Acto seguido se aplicó el Inventario Multiaxial de Personalidad MCMI-III de Theodore Millon, en su edición española (Cardenal y Sánchez, 2007) para el diagnóstico del trastorno histriónico de la personalidad. Luego, se llevó a cabo la entrevista la cual duró alrededor de 20 minutos. Estas entrevistas fueron grabadas en video, luego reproducidas y transcritas para la realización del análisis de contenido de cada entrevista. Fueron seleccionadas aquellas que reunieran en el mismo los criterios de suficiencia, pertinencia, claridad de contenido, lógica en un 95\% según acuerdo de los tres evaluadores independientes entrenados para el caso. Este proceso dejó 30 entrevistas por género en los grupos de casos y de controles. 


\section{Cuarta etapa}

Se tomó cada entrevista transcrita como la "unidad de contexto" tanto de los participantes del grupo de los casos como de los controles según la técnica de análisis de contenido (Bardin, 1996; Krippendorff, 1997, 2013). Se inició con la segmentación de párrafos y oraciones (unidad de análisis gramatical-unidad de registro). Se escogieron las palabras y grupos de palabras relacionadas con estimaciones, apreciaciones, valoraciones, estándares, así como la jerarquización de las mismas ya fuera en expresiones comparativas como motivacionales o prospectivas de la experiencia personal narrada y comentada por los entrevistados.

Se identificaron los temas (unidad de enumeración) (Esterberg, 2002), así como proposiciones relativas a las categorías o variedad de valores tanto en los relatos del grupo de los casos como de los controles. Se clasificaron los valores en un repertorio unificado que emergió en los casos en los dos géneros. Un grupo independiente de evaluadores retomó estos resultados del análisis de contenido y lo contrastó con las unidades de contextos fuentes para identificar concordancias o discrepancias. Aquellas que tuvieron discrepancias del 5\% fueron eliminadas. Con las restantes se realizó un procesamiento complementario con la herramienta ATLAS.ti ${ }^{\circledast}$ (Friese, 2013, 2014; Muhr \& Friese, 2004).

Se siguieron los criterios de Berelson (1967) para el análisis específico de categorías, tales como: homogeneidad, exhaustividad, exclusividad para evitar reclasificaciones categoriales, adecuación, pertinencia. Se utilizaron dos codificadores independientes por grupos de entrevistas para garantizar mayor objetividad y reproducibilidad, el nivel de acuerdo alcanzado fue del 95\% (Fox, 1981). Posteriormente se compararon los resultados obtenidos analizando agrupamientos de clase e intercalase (Coleman \& Unrau, 2005; ) resultando un repertorio de 12 valores característicos del grupo de los casos que no se observó en el grupo de controles 


\section{Quinta etapa}

Los 12 valores obtenidos del análisis de contenido fueron sometidos luego en otra entrevista individual a validación convergente tanto en los participantes del grupo de los casos como de los controles 2 meses después de las entrevistas, obteniéndose validación convergente significativa en los participantes del grupo de los casos y no en los controles. Una vez terminado este proceso se citó a cada uno de los participantes del estudio individualmente haciéndoles la devolución de la información para lo cual se asignaron dos psicólogos independientes al estudio que verificaron el proceso que se realizó con cada participante y que acompañó el proceso de cierre de información. A cada participante se le informó en detalle y de manera concreta sus resultados de evaluación, las características y vulnerabilidades de su condición, en el grupo de los casos, se repitió aquella información que no fuera comprensible para el participante y se dio atención durante dos sesiones más de 40 minutos cada una a todos ellos. Se los instó a permanecer en un proceso en los servicios de atención en salud mental de la red pública nacional de Colombia que no les acarrea ningún costo y se realizaron las diligencias para establecer los contactos para tal fin. A los participantes del grupo de controles, se les hizo una entrevista de devolución y evaluación de su estado post-estudio a cada uno con duración de 40 minutos con psicólogo. Se aclararon en los dos grupos todas las dudas que surgieron en los participantes.

\section{Resultados}

Los 12 valores característicos obtenidos del análisis de contenido se presentan en la tabla 2 , con sus respectivas proposiciones apreciativas, con las frecuencias en que fueron reportados como importantes en su experiencia personal, tanto en el grupo de los casos como en el de los controles. 


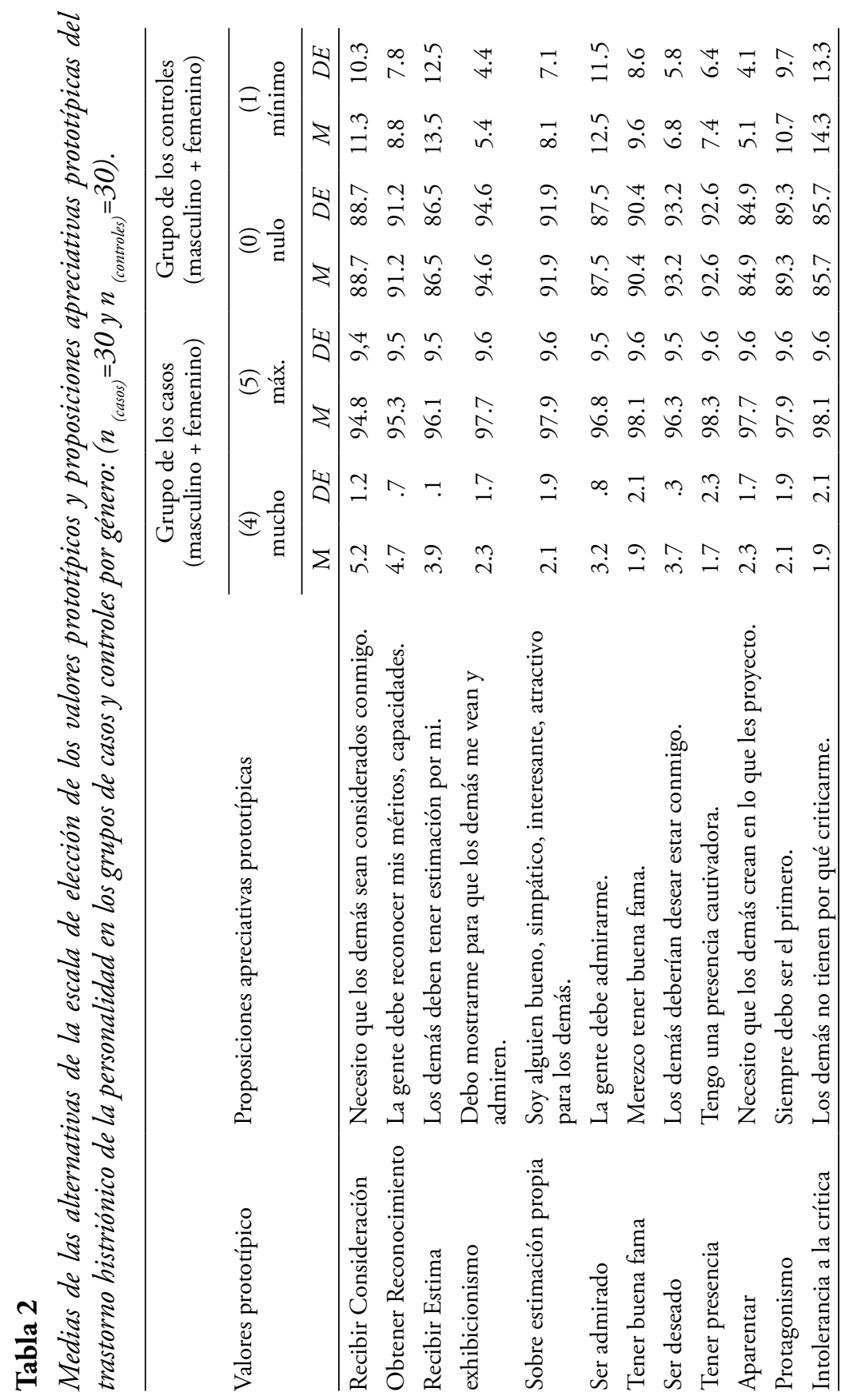


En la tabla 2 se muestran las medias y desviaciones de las elecciones de los valores y proposiciones apreciativas correspondientes, obtenidos del análisis de contenido, en una escala de 0 a 5 , en el grupo de los casos, en los dos géneros; $y$, de los controles, de igual manera. Como se aprecia las medias en el grupo de los casos (masculino y femenino) fueron más altas en las alternativas 4 y 5 ; y, en los controles (masculino y femenino) 0 y 1 , de igual manera, quedando sin ser escogidas las alternativas restantes según el caso (nulo $=0$, mínimo $=1$, algo $=2$; medio $=$ 3 , mucho $=4$, máximo $=5$ ). Esto indica que dichos valores fueron ratificados como propios de su experiencia de vida en los participantes con trastorno histriónico de la personalidad versus la no-identificación con ellos de los participantes sin el trastorno. De esta forma los valores fueron: Recibir consideración de los demás, obtener su reconocimiento y estima, el propio exhibicionismo y sobre estimación; el ser admirado y tener buena fama, ser deseado y tener presencia, aparentar, tener protagonismo e intolerancia a la crítica, todos en grado extremo.

Estos valores, por otra parte no fueron considerados como definitorios de su experiencia en los participantes del grupo de los controles. Este repertorio fue reaplicado 8 semanas después a los mismos participantes en sesión individual obteniéndose un $(\rho x x=.87)$ indicativo de la estabilidad temporal de los mismos (Anastasi, 1976; Magnusson, 1982).

\section{Tabla 3}

Valores $t$ de student para la diferencia de medias entre los valores $y$ proposiciones apreciativas del trastorno histriónico de la personalidad en los casos vs controles

\begin{tabular}{lccccccc}
\hline & $\begin{array}{c}t \\
\text { (obtenida) }\end{array}$ & $S_{1}{ }^{2}$ & $S_{1}{ }^{2}$ & $n_{1}$ & $n_{2}$ & $\begin{array}{c}t \\
\text { (esperada) }\end{array}$ & $g l$ \\
\hline Casos vs controles & 5.477 & .25 & .25 & 60 & 60 & $3.460^{*}$ & 180 \\
\hline$p<.001$ & & & & & & &
\end{tabular}


La tabla 3 muestra los valores obtenidos $t=5.477$ con $p \leq .01$ entre los casos vs los controles, con un $t=3.460$ esperado $(p \leq .01)$ y $g l=180$ que muestran que el primero, fue superior al segundo validándose de esta forma la hipótesis de investigación sobre la existencia de un repertorio de valores prototípicos del trastorno de la personalidad histriónica en el grupo de los casos a diferencia del grupo de los controles donde no se verificó dicha hipótesis, mediante la aplicación de la prueba t de student.

\section{Discusión}

Los resultados previos muestran que los puntajes en el grupo de casos son más altos en la escala respecto del grupo de los controles con lo cual se observa la importancia extrema que dichos valores tienen para el afectado. En ese sentido las situaciones que viven las personas con trastorno histriónico de la personalidad priorizan y magnifican cada uno de los doce valores prototípicos en si mismos, respecto de los demás, respecto de los diferentes ámbitos de su vida haciéndosela difícil de vivir en términos adaptativos.

Estos valores con los más altos puntajes en el grupo de los casos indican cualitativamente que quienes viven el trastorno histriónico de la personalidad valoran en forma superlativa su propia imagen, buscan la admiración de los demás hacia sus conductas, pensamientos y sentimientos a toda costa; igualmente jerarquizan en extremo su experiencia entorno al protagonismo, exhibicionismo, reconocimiento de los demás, así como una búsqueda de propósito narcisista caracterizada por la muy baja tolerancia a la frustración cuando no consigue sus propósitos. Esta fusión cognitiva es un aspecto de la problemática del cliente que reviste gran atención ya que esta determina la facilidad con la cual este puede tomar perspectiva de sí mismo respecto de su situación y de esta manera avanzar hacia un contexto situacional alternativo más flexible y adaptativo. Esto remarca la pertinencia de trabajar en la psicoterapia a partir del repertorio de valores prototípicos ya que la defusión 
cognitiva implica darse cuenta de cuán implicado está el cliente con sus sistema de valores, qué elementos del contexto acompañan dicha situación, y cómo puede tomar distancia conceptual y valorativa respecto de los mismos (Rehfeldt, Dillen, Ziomek \& Kowalchuk, 2007). Si se cuenta con una guía de valores específicos que puedan verse representados en los distintos ámbitos de la vida y de las relaciones consigo mismo y los demás apuntar hacia nuevas alternativas de valor se hace un ejercicio más orientado, más directivo.

El trabajo terapéutico de la ACT tiene así un eje de acción con en el cual enfocarse en el momento presente (Bishop et al., 2004; Dimidjian \& Linehan, 2003; Fletcher \& Hayes, 2005; Kabat-Zinn, 1994; Langer, 2000) tanto para el cliente como para el terapeuta descubriendo motivaciones para la acción y para la recuperación del dominio de la experiencia (Luciano, Rodríguez \& Gutiérrez, 2004). En este aspecto las verbalizaciones del cliente y terapeuta son muy importantes puesto que el lenguaje tiene un rol según el cual permite modificar las funciones de las experiencias privadas (pensamientos y sentimientos) y de las acciones a través del entrenamiento (Luciano, Gómez \& Rodríguez, 2007; Rehfeldt \& Barnes-Holmes, 2009), teniendo siempre en cuenta, por supuesto, el abordaje de la evitación experiencial (Tull, Gratz, Salters \& Roemer, 2004).

El desentramado de valores idiosincrásicos, su clarificación y reconocimiento se hace más localizado al existir un repertorio característico de estos valores, volviéndose así un baluarte técnico de relevancia en la ACT (Paéz, Gutiérrez, Valdivia \& Luciano, 2006). La validación obtenida de los mismos implica que dicha estructura evaluativa del trastorno puede ser rastreada, reconocida y objetivada de manera confiable. Esta validación convergente del repertorio de valores prototípicos del trastorno histriónico de la personalidad, mostró que la experiencia de dicho trastorno crea una inflexibilidad en el conjunto de valores que definen la naturaleza valorativa del mismo al estar muy jerarquizados, a la vez se encuentra que esta estructura valorativa constituye una red de estimaciones que guía la toma de decisiones y la solución de problemas a la manera idiosincrásica en que el trastorno mismo está constituido, es 
decir, la forma de afrontamiento está supeditada a dichos valores y en función de ellos se adquieren las motivaciones para la acción histriónica.

Se halló que sin distinción de edad, género, estado civil, nivel educativo o estrato socio económico el repertorio de valores prototípicos del trastorno histriónico de la personalidad fue confirmado.

Dentro de las limitaciones de este estudio se encuentran los grupos etarios con los cuales se trabajó ya que es importante contar con muestras con edades mayores y menores a las del presente estudio, por otra parte es importante ver el contraste entre muestras de la zona urbana y rural ya que no pudimos en este estudio acceder a esta última.

A partir de este estudio se espera que surjan otros en otras poblaciones con diferentes características geográficas, étnicas, culturales y socio económicas que hagan una masa crítica de hallazgos que develen la existencia de estos valores no solo en este sino también en otros trastornos de la personalidad. Este inventario, solo cumple una función orientativa para evitar divagaciones en el proceso terapéutico que se presentan en la práctica de la ACT en estos casos, en especial cuando los propios clientes se encuentran especialmente confundidos respecto de lo que les pasa y no se logran focalizar.

Se recomienda explorar estos repertorios en poblaciones con otros trastornos de la personalidad con el objetivo de hacer validaciones divergentes que confirmen o desconfirmen lo aquí hallado teniendo en cuenta que los TP y en especial el TPH se encuentran poco estudiados y atendidos en general tanto en la investigación como en la clínica.

\section{Referencias}

Addy, C. (2013). Identifying your values. Recuperado de https:// myjourneywithdepression.wordpress.com/2013/09/10/ act-identifying-your-values/

Anastasi, A. (1976). Psychological Testing. New York: Macmillan. Addis, M. E. \& Jacobson, N. S. (1996). Reason-giving and the process and outcome of cognitive-behavioral psychotherapies. Journal of 
Consulting and Clinical Psychology, 64, 1417-1424. https://doi. org/10.1037/0022-006X.64.6.1417

Bach, P. \& Hayes, S. C. (2002). The use of Acceptance and Commitment Therapy to prevent the rehospitalization of psychotic patients: A randomized controlled trial. Journal of Consulting and Clinical Psychology, 70(5), 1129-1139. https://doi. org/10.1037/0022-006X.70.5.1129

Bardin, L. (1996) Análisis de contenido. Madrid: Akal Ediciones.

Bishop, S. R., Lau, M., Shapiro, S., Carlson, L., Anderson, N. D. Carmody, J. \& Devins, G. (2004). Mindfulness: A proposed operational definition. Clinical Psychology:Science and Practice, 11, 230-241. https://doi.org/10.1093/clipsy.bph077

Bond, F. W. \& Bunce, D. (2000). Mediators of change in emotionfocused and problem-focused worksite stress management interventions. Journal of Occupational Health Psychology, 5, 156163. https://doi.org/10.1037/1076-8998.5.1.156

Cardenal, V. \& Sánchez, M.P. (2007). Adaptación y baremación al español del Inventario Clínico Multiaxial de Millon-III (MCMIIII). Madrid: TEA, Ediciones.

Cohen, G. L., Garcia, J., Apfel, N. \& Master, A. (2006). Reducing the racial achievement gap: A social-psychological intervention. Science, 313, 1307-1310. https://doi.org/10.1126/ science. 1128317

Coleman, H. \& Unrau, Y. A. (2005). Analyzing qualitative data. En R. M. Grinnell e Y. A. Unrau (Eds.), Social work: Research and evaluation: Quantitative and qualitative approaches (7a. ed.) (pp. 403-420). Nueva York: Oxford University Press.

Congreso de la República de Colombia. Ley 142 de 1994 Por la cual se establece el régimen de los servicios públicos domiciliarios y se dictan otras disposiciones. Recuperado de https://comision6senado.files.wordpress.com/2013/03/ley-142-de-1994.pdf

Cloninger, C.R. (2000). A practical way to diagnosis personality disorders. Journal of Personality Disorders, 14, 99-108. https://doi. org/10.1521/pedi.2000.14.2.99 
Dahl, J., Wilson, K. G., Luciano, C. \& Hayes, S. C. (2005). Acceptance and Commitment Therapy for Chronic Pain. Reno, NV: Context Press.

DANE (2011). Infraestructura Colombiana de datos. http://190.25.231. 246:8080/Dane/tree.jsf

Departamento Nacional de Planeación de Colombia (2005). Documento 3386 del Consejo Nacional de Politica Económica y Social. Recuperado de http://www.dnp.gov.co/PortalWeb/Portals/0/ archivos/documentos/Subdireccion/Conpes/3386.pdf

Dimidjian, S. D. \& Linehan, M. M. (2003). Mindfulness practice. En W. O'Donohue, J. Fisher y S. Hayes (Eds.), Cognitive behavior therapy: Applying empirically supported techniques in your practice (pp. 229-237). New York: Wiley.

Echeburúa, E. \& Fernández-Montalvo, J. (2007). Male batterers with and without psychopathy: An exploratory study in Spanish prisons. International Journal of Offender Therapy and Comparative Criminology, 51, 254-263. https://doi. org/10.1177/0306624X06291460

Echeburúa, E., Fernández-Montalvo, J. \& Amor, P. J. (2006). Psychological treatment of men convicted of gender violence: A pilot-study in Spanish prisons. International Journal of Offender Therapy and Comparative Criminology, 50, 57-70. https://doi. org/10.1177/0306624X05277662

Eifert, G. \& Forsyth, J. (2005). Acceptance and Commitment Therapy for anxiety disorders. Oakland, CA: New Harbinger.

Eifert, G. H., McKay, M. \& Forsyth, J. P. (2006). ACT on life not on anger: The new Acceptance and Commitment Therapy guide to problem anger. Oakland, CA: New Harbinger.

Esbec, E. \& Echeburúa, E. (2011). La reformulación de los trastornos de la personalidad en el DSM-V. Actas Españolas de Psiquiatría, 39, 1-11.

Esbec, E. \& Robles J. L. (2013). Evaluación de los trastornos de la personalidad en Psiquiatría Forense. En S. Delgado y J. M. Maza (Eds.), Psiquiatría Legal y Forense (pp. 141-178). Barcelona: Bosch. 
Esterberg, K. G. (2002). Qualitative methods in social research. Nueva York, NY: McGraw-Hill.

Fernández-Montalvo, J. \& Echeburúa, E. (2008). Trastornos de personalidad y psicopatía en hombres condenados por violencia grave contra la pareja. Psicothema, 20, 193-198.

Fletcher, L. \& Hayes, S. C. (2005). Relational frame theory, acceptance and commitment therapy, and a functional analytic definition of mindfulness. Journal of Rational Emotive and Cognitive Behavioral Therapy, 23, 315-336. https://doi.org/10.1007/ s10942-005-0017-7

First, M. (1999). SCID-II: entrevista clinica estructurada para los trastornos de la personalidad del eje II del DSM-IV.Guia del usuario. Entrevista clínica. Cuestionario de personalidad. Masson.

First, M. (2001). DSM-IV-TR: Manual de Diagnóstico y Estadístico de los Trastornos Mentales. Barcelona: Masson.

Fleiss, J.L. (1971).Measuring nominal scale agreement among many raters. Psychology Bulletin, 76, 378-382. https://doi.org/10.1037/ h0031619

Follette, C. \& Pistorello, J. (2007). Finding life beyond trauma: Using acceptance and commitment therapy to heal from post-traumatic stress and trauma-related problems. Oakland, CA: New Harbinger.

Fox, D.J. (1981). El proceso de investigación en educación. Pamplona, EUNSA.

Frances, A., Pincus, H. \& First, M. (2008). Manual diagnóstico y estadistico de los trastornos mentales. Madrid: Masson.

Friese, S. (2013). ATLAS.ti 7 User Manual. Berlin, Alemania: Scientific Software Development. Recuperado de http://atlasti.com/ manual.html

Friese, S. (2014). Qualitative Data Analysis with ATLAS.ti. London: Sage. Gallagher, R. P. (2009). National survey of counseling center directors. Alexandria, VA: International Association of Counseling Services.

Gifford, E. V., Kohlenberg, B. S., Hayes, S. C., Antonuccio, D. O., Piasecki, M. M.,Rasmussen-Hall, M. L.\& Palm, K. M. (2004). Acceptance theory-based treatment for smoking cessa- 
tion: An initial trial of Acceptance and Commitment Therapy. Behavior Therapy, 35, 689-705. https://doi.org/10.1016/ S0005-7894(04)80015-7

Giner, S. (1975). Análisis. En VV.AA., Diccionario de Ciencias Sociales. Madrid: Instituto de Estudios Políticos.

Gutiérrez-Zotes, J.A., Bayón, C., Montserrat, C., Valero, J., Labad, A. \& Cloninger C. R. (2004). Inventario del Temperamento y el CarácterRevisado (TCI-R). Baremación y datos normativos en una muestra de población general. Actas Españolas de Psiquiatría, 32, 8-15.

Gregg, J. A., Callaghan, G. M., Hayes, S. C. \& Glenn-Lawson, J. L. (2007). Improving diabetes self-management through acceptance, mindfulness, and values: A randomized controlled trial. Journal of Consulting and Clinical Psychology, 75, 336-343. https://doi.org/10.1037/0022-006X.75.2.336

Hayes, S. C., Barnes-Holmes, D. \& Roche, B. (Eds.). (2001). Relational Frame Theory: A Post-Skinnerian account of human language and cognition. New York: Plenum Press.

Hayes, S. C., Luoma, J., Bond, F., Masuda, A. \& Lillis, J. (2006). Acceptance and Commitment Therapy: Model, processes, and outcomes. Behaviour Research and Therapy, 44, 1-25. https:// doi.org/10.1016/j.brat.2005.06.006

Hayes, S. C., Strosahl, K. \& Wilson, K. G. (1999). Acceptance and Commitment Therapy: An experiential approach to behavior change. New York: Guilford Press.

Kabat-Zinn, J. 1994. Wherever you go there you are: Mindfulness meditations in everyday life. New York: Hyperion.

Kashdan, T. B., Barrios, V., Forsyth, J. P.\& Steger, M. F. (2006). Experiential avoidance as a generalized psychological vulnerability: Comparisons with coping and emotion regulation strategies. Behaviour Research and Therapy, 44, 1301-1320. https://doi. org/10.1016/j.brat.2005.10.003

Krippendorf, K. (1997). Metodología de análisis de contenido. Teoría y práctica. Barcelona: Paidós. 
Krippendorff, K. (2013). Content analysis: An introduction to its methodology (3rd ed.). Thousand Oaks, CA: Sage.

Langer, E. J. (2000). Mindful learning. Current Directions in Psychological Science,9, 220-223. https://doi.org/10.1111/1467-8721.00099 López-Ibor, J.J., Pérez Urdániz, A. \& Rubio, V. (1996). Examen internacional de los trastornos de personalidad. Madrid: Méditor.

López-Pérez, B., Fernández-Pinto, I. \& Abad, F. J. (2008). Test de empatía cognitiva y afectiva. Madrid: Ediciones TEA.

Luciano, M. C., Gómez, I. \& Rodríguez, M. (2007). The role of multiple-exemplar training and naming in establishing derived equivalence in an infant. Journal of the Experimental Analysis of Behavior, 87, 349-365.

Luciano, M.C., Rodríguez, M. \& Gutiérrez, O. (2004). A proposal for synthesizing verbal contexts in Experiential Avoidance Disorder and Acceptance and Commitment Therapy. International Journal of Psychology and Psychological Therapy, 4, 377-394. https://doi.org/10.1901/jeab.2007.08-06

Luoma, J., Hayes, S. C. \& Walser, R. (2007). Learning ACT. Oakland, CA: New Harbinger.

Marx, B. P. \& Sloan, D. M. (2002). The role of emotion in the psychological functioning of adult survivors of childhood sexual abuse. Behavior Therapy, 33, 563-577. https://doi.org/10.1016/ S0005-7894(02)80017-X

Magnusson D. (1982). Teoría de los tests. México: Trillas.

Millon, T. \& Davis, R. (1998). Trastornos de personalidad. Más allá del $D S M-I V$. Barcelona: Masson.

Muhr, T. \& Friese, S. (2004). User's manual for ATLAS.ti 5.0 (2a. ed.). Berlin, Alemania: Scientific Software Development. Recuperado de http://atlasti.com/manual.html

Paéz, M., Gutiérrez, O., Valdivia, S. \& Luciano, C. (2006). Terapia de Aceptación y Compromiso (ACT) y la importancia de los valores personales en el contexto de la terapia psicológica. International Journal of Psychology and Psychological Therapy, 6(1), 1-20. 
Rehfeldt, R. A. \& Barnes-Holmes, Y. (Eds.). (2009). Applied programs for derived relational responding. Oakland, CA: New Harbinger Rehfeldt, R. A., Dillen, J. E., Ziomek, M. M. \& Kowalchuk, R. K. (2007). Assessing relational learning deficits in perspective-taking in children with high-functioning autism spectrum disorder. Psychological Record, 57, 23-47. https://doi.org/10.1007/ BF03395563

Rosenthal, M. Z., Rasmussen-Hall, M. L., Palm, K. M., Batten, S. V. \& Follette, V. M. (2005). Chronic avoidance helps explain the relationship between severity of childhood sexual abuse and psychological distress in adulthood. Journal of Child Sexual Abuse, 14, 25-41. https://doi.org/10.1300/J070v14n04_02

Skodol A.E, Bender, D.S., Oldham, J.M., Clark, L.A., Morey, L.C., Verheul, R., Krueger, R.F. \& Siever L.J. (2011). Proposed changes in personality and personality disorder assessment and diagnosis for DSM-5 Part II: clinical application. Journal of Personality Disorders, 7, 23-40. https://doi.org/10.1037/a0021892

Spitzer, R.L., Williams, J.B. \& Gibbon, M. (1989). Structured Clinical Interview for DSM-III-R, Axis II. SCID-II. New York, NY: York State Psychiatric Institute.

Tull, M. T., Gratz, K. L., Salters, K. \& Roemer, L. (2004). The role of experiential avoidance in posttraumatic stress symptoms and symptoms of depression, anxiety, and somatization. Journal of Nervous o Mental Disease, 192(11), 754-761. https://doi. org/10.1097/01.nmd.0000144694.30121.89

Twohig, M. P., Hayes, S. C., Plumb, J. C., Pruitt, L. D., Collins, A. B., Hazlett- Stevens, H. \& Woidneck, M. R. (2010). A randomized clinical trial of Acceptance and Commitment Therapy vs. Progressive Relaxation Training for obsessive compulsive disorder. Journal of Consulting and Clinical Psychology, 78, 705-716. https://doi.org/10.1037/a0020508

Twohig, M. P., Shoenberger, D. \& Hayes, S. C. (2007). A preliminary investigation of Acceptance and Commitment Therapy as a treatment for marijuana dependence in adults. Journal of 
Applied Behavior Analysis, 40, 619-632. https://doi.org/10.1901/ jaba.2007.619-632

Villatte, M., Monestes, J. L., McHugh, L., Freixa i Baqué, E. \& Loas, G. (2008). Assessing deictic relational responding in social anhedonia: A functional approach to the development of Theory of Mind impairments. International Journal of Behavioral Consultation and Therapy, 4, 360-373. https://doi.org/10.1037/h0100867 Wicksell, R. K., Ahlqvist, J., Bring, A., Melin, L. \& Olsson, G. L. (2008). Can exposure and acceptance strategies improve functioning and quality of life in people with chronic pain and whiplash associated disorders (WAD)? A randomized controlled trial. Cognitive Behaviour Therapy, 37, 1-14. https://doi. org/10.1080/16506070802078970

Wicksell, R. K., Renofalt, J., Olsson, G. L., Bond, F. W. \& Melin, L. (2008). Avoidance and cognitive fusion-central components in pain related disability? Development and preliminary validation of the Psychological Inflexibility in Pain Scale (PIPS). European Journal of Pain, 12(4), 491-500. https://doi.org/10.1016/j. ejpain.2007.08.003

Zettle, R. (2007). ACT for depression: A clinician's guide to using Acceptance \& Commitment Therapy in treating depression. Oakland, CA: New Harbinger.

Zettle, R. D., Rains, J. C. \& Hayes, S. C. (2011). Processes of change in Acceptance and Commitment Therapy and Cognitive Therapy for depression: A meditational reanalysis of Zettle and Rains (1989). Behavior Modification, 35, 265-283. https://doi. org/10.1177/0145445511398344

Weltzler, S. (1990). The Millon Clinical Multiaxial Inventory (MCMI): A review. Journal of Personality Assessment, 55, 445-464. https:// doi.org/10.1080/00223891.1990.9674083

Recibido: 15 de octubre, 2016

Revisado: 2 de octubre, 2017 Aceptado: 12 de octubre, 2017 


\section{Apéndice}

\section{Entrevista estructurada de valores asociados al trastorno histriónico de la personalidad.}

\section{Primera parte}

¿Cuáles son sus prioridades: ser reconocido, admirado, respetado halagado, u otras?, ¿cuáles?; ¿Qué es lo que vale más para Ud. en la vida”?; ¿Qué cosas esperaría que produjeran en los demás respecto a Ud., reconocimiento, admiración, fama, interés?; ¿Cuáles son sus "no puedo soportarlo", "no aguanto más" en su vida?; ¿Hacia qué experimenta los mayores sentimientos de frustración, desengaño, decepción, impotencia?

\section{Segunda parte}

Constituida por preguntas abiertas a ser contestadas por el participante:

Cuando se trata de tomar una posición y una decisión en una situación en la que Usted es ignorado, suplantado, desestimado, no correspondido, ¿qué sentimientos genera en Ud., qué tan intensos son?, ¿Qué tan frecuentemente le sucede?, ¿Cuánto le suelen durar estos sentimientos?, ¿Cómo afectan dichos sentimientos su tranquilidad, su equilibrio y sus relaciones con los demás?

$\mathrm{Al}$ tener gente a su alrededor, ¿qué es lo que más le importa respecto a ellos y Ud.?

Cuando otros se oponen a lo que Usted dice, hace o quiere, ¿¿A qué le da más importancia, cuál es su posición?, ¿Por qué?

Cuando los demás son desconsiderados, abusivos, indecorosos con Ud., ¿qué es lo que más le perturba de ello?

A qué suele dar más prioridad: ¿a lo que digan los demás, a su honra, su fama o a otras cosas?, ¿Cuáles?

¿Qué estimación da a la incondicionalidad de los demás?

¿Qué tan importante es para ud que las personas sean delicadas, detallistas, consideradas?

¿Qué lugar tiene en su vida "ser reconocido, ser tenido en cuenta, ser estimado por otros? 